\title{
La relación entre la filosofía educativa del profesorado de guitarra de las Escuelas de música y la motivación de su alumnado
}

\author{
Yolanda Sierra-Hernández ${ }^{1}$; Cristina Arriaga-Sanz ${ }^{2}$; José Mª Madariaga-Orbea ${ }^{3}$
}

Recibido: 13 de enero de 2019 / Aceptado: 6 de noviembre de 2019

Resumen. En esta investigación se analiza la relación entre la forma de entender la educación del profesorado de guitarra de las Escuelas de música y la motivación de su alumnado. Tomando como referencia a Parkinson (2016), se proponen dos perfiles educativos del profesorado: uno centrado en la innovación y el disfrute y otro en la tradición y el dominio. Los resultados muestran que, el alumnado de los docentes innovadores enfocados al disfrute, tiene mayor motivación y una relación más próxima con sus educadores que el del otro tipo de docentes. De estos resultados se derivan implicaciones para la formación del profesorado.

Palabras clave: Educación musical; Escuelas de música; motivación; guitarra; relación educativa.

\section{[en] The relationship between the educational philosophy of the Music schools' guitar teachers and the motivation of their students}

Abstract. This research analyzes the relationship between the way the guitar teachers of Music schools understand the teaching and the motivation of their students. Taking Parkinson (2016) as a reference, two educational profiles of the teaching staff are proposed: one focused on innovation and enjoyment and the other on tradition and mastery. The results show that the students of innovative teachers focused on enjoyment have a greater motivation and a closer relationship with their educators than the students of the other types of teachers. Several implications for the teacher's education are derived from these results.

Keywords: Musical education; Music schools; motivation; guitar; educational relationship.

Sumario. 1. Introducción. 2. El perfil del profesorado de música. 3. La motivación en educación musical. 4. Metodología. 4.1. Hipótesis 4.2. Participantes 4.3. Instrumentos de medida 4.5. Procedimiento. 5. Resultados. 5.1. Caracterización del profesorado. 5.2. Motivación del alumnado. 6. Conclusiones. 7. Referencias bibliográficas.

Cómo citar: Sierra-Hernández, Y.; Arriaga-Sanz, C.; Madariaga-Orbea, J.M. (2020) La relación entre la filosofía educativa del profesorado de guitarra de las Escuelas de música y la motivación de su alumnado, en Revista Electrónica Complutense de Investigación en Educación Musical, 17, 73-84

\section{Introducción}

Este estudio es parte de una investigación que surge de la necesidad de indagar acerca de las razones que llevan al alumnado de guitarra de las escuelas de música a mantener el interés por el estudio. De acuerdo con Cain (2013), las razones que llevan al estudiante a aprender a tocar un instrumento son variadas y no responden, esencialmente, a ambiciones profesionales.

Universidad del País Vasco (España)

E-mail: josetxu.madariaga@ehu.eus 
Además, si no sienten que las clases responden a sus expectativas pueden dejar de asistir a ellas, de modo que el reto del profesorado será identificar e intentar dar respuesta a las necesidades de los estudiantes. Es por ello evidente el interés por conocer aquello que hace que este alumnado esté motivado para continuar estudiando.

Respecto a los diferentes factores intervinientes en la motivación del alumnado de guitarra, se ha determinado que esta investigación se centrará en analizar el posible rol que pueden desempeñar el modo de relacionarse con su profesorado, la forma de entender la educación de dichos docentes. Se entiende que el profesorado es el encargado de desarrollar estrategias para acercarse al alumnado y crear el vínculo afectivo que haga que esta relación facilite la motivación hacia el aprendizaje. En este sentido, el rol del profesorado es fundamental para abordar las tareas con sus estudiantes, ya que estaría relacionado con su motivación (Arriaga y Madariaga, 2014; Campayo y Cabedo, 2018; Madariaga y Arriaga, 2011).

En las escuelas de música, la enseñanza es principalmente individual, por lo que la interacción entre profesorado y alumnado es constante (López, 2016). De hecho, Torrado y Pozo (2008) apuntan que "la principal ventaja de esta enseñanza sea, posiblemente, el contacto directo con el alumno" (p 4). En este sentido, la relación que se da entre docentes y discentes no se basa únicamente en la técnica utilizada, sino también en el clima emocional y la interacción que se genera (Widad, 2013). Esto es debido a que las escuelas de música surgen como respuesta a la demanda social de todo tipo de personas que quieren hacer música, sin alcanzar el grado de profesionalidad y rigor académico de los conservatorios.

Como consecuencia, el aprendizaje de la música se hace, principalmente, a través del tutor o tutora de instrumento en su clase individual. Por ello, las escuelas de música se insertan en el grupo de las denominadas enseñanzas especializadas, que poseen unas características específicas:

1. Establecimiento de una relación personal entre alumnado y profesorado en la que, de forma opcional, según las características del centro y del profesorado mismo, van a influir las emociones y los afectos.

2. Diferencias de edad y tipología del alumnado que obligan a ofrecer un planteamiento educativo flexible para adaptarse a sus diferentes necesidades.

Ahora bien, a pesar de la importancia que las escuelas de música otorgan a su profesorado por estar bien formado (De Alba, 2016), se debe tener en cuenta que una parte significativa de este colectivo ha tenido que reciclarse, ya que viene de impartir clases en conservatorios que son centros que tratan de responder a otro tipo de necesidades. Esta circunstancia, en ocasiones, no favorece el avance y la adaptación a las necesidades de las escuelas de música actuales.

Además, Morant (2013) previene acerca de algunas carencias formativas de base en el profesorado de las escuelas de música, ya que tanto el título profesional de estudios musicales como el superior, no tienen en muchos casos un perfil docente. Por tanto, no preparan para la salida profesional que representan las plazas de profesorado en las escuelas de música, en las que es necesario adecuar la programación de la enseñanza a los intereses, dedicación y ritmo de aprendizaje del alumno (Decreto 289/1992, art. 1).

Es por ello que, este estudio pretende analizar si la tipología del profesorado según su perfil educativo, está relacionada con la motivación de su alumnado. Es decir, si un perfil docente se relaciona en mayor o menor medida con la motivación y de qué manera.

\section{El perfil del profesorado de música}

Diversos estudios indagan acerca de las concepciones y prácticas del profesorado de enseñanza instrumental (Jorquera, 2002; Parkinson, 2016; Pozo, Bautista y Torrado, 2008). En concreto, Parkinson (2016), reflexiona sobre su propia experiencia como profesor de guitarra, en relación con la necesidad de combinar de forma equilibrada el progreso en el aprendizaje musical y el disfrute de cada estudiante. Subraya que su objetivo como maestro es fomentar el disfrute frente a la técnica a la hora de aprender a tocar un instrumento. Argumenta, por ejemplo, que muchos intérpretes de guitarra eléctrica no tienen cultura musical, ni siguen unos estándares y normas establecidos pero, sin embargo, componen su propia música, improvisan, experimentan y disfrutan.

Ciertamente éste es un tema relevante en el ámbito de la educación musical, dado que el profesorado se encuentra habitualmente ante el reto de asegurar que sus estudiantes progresen en habilidades técnicas, al tiempo que disfrutan de la experiencia musical, ya que progreso y disfrute son complementarios (Hallam y Creech 2010; Parkinson, 2016). Ahora bien, en la práctica diaria el profesorado de música debe decidir sobre cuál de estas dos posibilidades prioriza, teniendo en cuenta que el predominio de una puede ir en detrimento de la otra.

En efecto, si se presta demasiada atención al desarrollo de la técnica, los estudiantes pueden aburrirse y desmotivarse y si se centra en el disfrute, el progreso puede bloquearse (Parkinson 2016). Partiendo de esta premisa, el estudio de Parkinson (2016) es relevante para nuestra investigación porque diseña un modelo que ayuda a caracterizar al profesorado de guitarra según su perfil educativo, en el que el dominio y la tradición van de la mano, así como la innovación y el disfrute. Por ello, y a partir de ahora, hablaremos de dos perfiles de profesorado; el centrado en la innovación y el disfrute y el que hace más hincapié en la tradición y el dominio. No obstante, queremos recalcar que el progreso y el disfrute no son mutuamente excluyentes (Hallam \& Creech, 2010), y de hecho son a menudo com- 
plementarios, aunque en ocasiones también pueden ser opuestos. Se trata de reflexionar sobre determinados aspectos que el profesorado puede tener en cuenta en su desarrollo docente, en cuanto a sus objetivos y valores, para atender a las necesidades motivacionales del alumnado, pero en ningún caso es nuestra intención hacer una clasificación rígida del profesorado ni polarizar dos tipos de enseñanza instrumental. En la práctica, el profesorado de música irá tomando decisiones de prioridad (lo uno o lo otro), ya que lo excesivo de uno puede ser en detrimento del otro. Si se presta demasiada atención al desarrollo técnico de los estudiantes, estos pueden sentirse desmotivados. En el otro, donde se da demasiada atención al disfrute de los estudiantes, el progreso puede bloquearse.

Con el fin de caracterizar dichos perfiles, se presentan a continuación los rasgos que describe Parkinson (2016) en su estudio:

Tabla 1. Perfiles del profesorado basados en la propuesta de Parkinson (2016)

\begin{tabular}{|l|l|l|l|}
\hline \multicolumn{1}{|c|}{ DOMINIO } & \multicolumn{1}{|c|}{ DISFRUTE } & \multicolumn{1}{c|}{ TRADICIÓN } & \multicolumn{1}{c|}{ INNOVACIÓN } \\
\hline $\begin{array}{l}\text { Do.1.Importancia de la } \\
\text { técnica }\end{array}$ & $\begin{array}{l}\text { Di.1.Importancia del dis- } \\
\text { frute }\end{array}$ & $\begin{array}{l}\text { T.1.Importancia de la tra- } \\
\text { dición }\end{array}$ & $\begin{array}{l}\text { I.1.Importancia de la inno- } \\
\text { vación }\end{array}$ \\
\hline $\begin{array}{l}\text { Do.2.Repertorio para desa- } \\
\text { rrollar la técnica }\end{array}$ & $\begin{array}{l}\text { Di.2.Repertorio para desa- } \\
\text { rrollar el disfrute }\end{array}$ & $\begin{array}{l}\text { T.2.Repertorio basado en } \\
\text { imitación y repetición }\end{array}$ & $\begin{array}{l}\text { I.2.Repertorio para desarro- } \\
\text { llar la imaginación, impro- } \\
\text { visación, creación }\end{array}$ \\
\hline $\begin{array}{l}\text { Do.3.Importancia de seguir } \\
\text { normas y estándares }\end{array}$ & $\begin{array}{l}\text { Di.3.Importancia de valorar } \\
\text { al alumnado y sus necesi- } \\
\text { dades }\end{array}$ & $\begin{array}{l}\text { T.3.Compromiso de cuidar } \\
\text { y promocionar el patrimo- } \\
\text { nio musical }\end{array}$ & $\begin{array}{l}\text { I.3.Seleccionar el repertorio } \\
\text { de manera colaborativa con } \\
\text { el alumnado }\end{array}$ \\
\hline $\begin{array}{l}\text { Do.4.El dominio es el obje- } \\
\text { tivo último de la enseñanza } \\
\text { instrumental. }\end{array}$ & $\begin{array}{l}\text { Di.4.El disfrute de la músi- } \\
\text { ca es el objetivo y parte de } \\
\text { la relación entre profesora- } \\
\text { do y alumnado. }\end{array}$ & $\begin{array}{l}\text { T.4.El objetivo está centra- } \\
\text { do en el talento. }\end{array}$ & $\begin{array}{l}\text { I.4.El objetivo es descubrir } \\
\text { los estilos adecuados al } \\
\text { alumnado. }\end{array}$ \\
\hline
\end{tabular}

\section{La motivación en educación musical}

En los últimos años, la motivación en educación musical se está investigando desde diferentes perspectivas (Austin, Renwick y McPherson, 2006; Hallam, 2002; McPherson y McCormick, 1999; McPherson y O’Neill, 2010; Renwick y McPherson, 2002; West, 2013; Triptana, 2016). En concreto, este estudio se centra como hemos podido ver en aquella perspectiva que la relaciona con el tipo de interacción entre alumnado y profesorado en el aula (Arriaga y Madariaga, 2014; Madariaga y Arriaga, 2011). En esta línea, Campayo y Cabedo (2018) afirman que la relación de confianza entre ambos aparece como un aspecto fundamental para favorecer la motivación del alumnado. Además, consideran que hay una mayor motivación del estudiante cuando percibe que el docente le apoya en su proceso de aprendizaje, en la medida que le hace sentirse más capaz para realizar la tarea.

En efecto, el papel del profesorado es fundamental para que el alumnado establezca su criterio de éxito o fracaso en función de la forma que tiene de relacionarse con él. Una relación adecuada entre ellos puede favorecer, tanto la motivación del estudiante como su persistencia en el estudio de la música (Arriaga y Madariaga, 2014; Campayo y Cabedo, 2018). Estrategias como ayudar al alumnado, reconocer su trabajo, animarle y ofrecerle su confianza promoviendo una relación cercana, corregir los errores basándose en sus virtudes o permitir que tenga autonomía en la elección de contenidos, influyen positivamente en la motivación (Madariaga y Arriaga, 2011; Campayo y Cabedo, 2018). De hecho, Arends (2007) sostiene que la motivación se relaciona de forma muy estrecha con el clima del aula resultante de la interacción entre profesorado y alumnado.

Por su parte Evans (2015) en su enfoque teórico de la autodeterminación para abordar la motivación en la educación musical conecta también con estas ideas. Este enfoque sostiene que el aprendizaje de la música es más motivador en un entorno social que satisface tres necesidades psicológicas básicas: competencia, relación y autonomía. Las experiencias de competencia y logro tienen una influencia motivadora, mientras que las de excesiva dificultad e incapacidad frustran la necesidad de competencia, lo que lleva a sentimientos de ineficacia. La relación también puede jugar un papel importante. En las primeras etapas del aprendizaje de la música, la seguridad de una relación cercana con el profesorado puede proporcionar una base importante para un enfoque posterior más centrado en la competencia y el dominio. Los ambientes que apoyan la relación son aquellos que proporcionan calidez y fomentan la conexión con el otro. Evans, McPherson y Davidson (2013, p. 609) encontraron referencias sobre las características del profesorado y su influencia en el disfrute del aprendizaje de la música, encontrando casos de estudiantes que abandonaron los estudios musicales porque el docente se "enfada porque no estamos interpretando de acuerdo con su estándar" porque "no estaba disfrutando la experiencia musical debido al director de banda" o porque "mi maestra y yo no nos llevábamos bien. Simplemente, dejé de disfrutar."

En lo que se refiere a la autonomía, Evans (2015) afirma que tradicionalmente el profesorado de música no ha apoyado la autonomía del alumnado en sus prácticas docentes y que, especialmente en conservatorios, puede ser exi- 
gente, e incluso en ocasiones perfeccionista y controlador. No obstante, De Bézenac \& Swindells (2009), compararon estudiantes de música clásica con estudiantes de música folklórica y popular y encontraron que estos últimos afirmaron experimentar más placer al participar en actividades musicales que los de música clásica. MacIntyre y Potter (2014) por su parte, compararon a estudiantes de guitarra con estudiantes de piano, encontrando que los de guitarra tenían más voluntad de tocar su instrumento y más autonomía que los de piano. En estos estudios los investigadores atribuyeron esta diferencia al estilo formal y rígido de la enseñanza de piano en comparación con el estilo más relajado y amigable/cercano de la enseñanza de la guitarra. En este sentido, Cain (2013) indica que las razones y las necesidades de los estudiantes de instrumento son muy variadas, y es tarea del profesorado identificar y dar respuesta a las mismas para reforzar su motivación.

Por su parte, Arriaga y Madariaga (2014) y Madariaga y Arriaga (2011), dentro de un contexto constructivista, otorgan gran importancia a la hora de entender la motivación a las pautas interactivas que se establecen entre profesorado y alumnado. Destacan la importancia de que la tarea a realizar tenga algún sentido para el alumnado, de forma que puedan percibirlo como algo atrayente y asequible para que se puedan implicar en ella. En este sentido, Renwick y McPherson (2002) consideran que una buena forma de motivar al alumnado es que este elija su repertorio. Asmus (1993) no obstante, advierte que un estudio demasiado profundo de una pieza musical, aunque sea muy atractiva para el alumnado, puede reducir su carácter motivador.

Burwell, Pickup y Young (2003) señalan que, a pesar de todo, durante muchos años el rol del docente de instrumento, ha consistido en ser modelo y autoridad acerca de lo que debe saber un instrumentista y la forma que tiene que interpretar determinado repertorio. Sin embargo, hay estudios que demuestran que es necesario romper con el enfoque del docente como modelo y explorar estilos de enseñanza más centrados en el alumnado. En este sentido, Mackwoth-Young (1990) subraya que la motivación de los estudiantes de piano puede aumentar si las clases están centradas en el alumnado, desarrollándose una mayor comunicación con el profesorado. Del mismo modo, McPhail (2010) también aboga, en relación con la motivación, por una enseñanza de la música más holística, en la que la interacción entre profesorado y alumnado esté dirigida a desarrollar la autonomía y el pensamiento crítico. Triptana (2016) subraya incluso que, establecer un clima afectivo y estimulante que favorezca el proceso de enseñanza-aprendizaje, es uno de los aspectos clave para mejorar la motivación.

En definitiva, los dos objetivos principales de esta investigación son: a) establecer la posible relación existente entre la motivación del alumnado de guitarra de las Escuelas de Música y, la forma de relacionarse dicho alumnado con su profesorado, y b) analizar la posible relación de la motivación de dicho alumnado con el perfil educativo del profesorado correspondiente (Parkinson, 2016).

\section{Metodología}

\subsection{Hipótesis}

- Hipótesis 1. Existen dos perfiles diferentes de profesorado en función de su filosofía acerca de la educación musical, uno centrado en la innovación y el disfrute y otro que hace más hincapié en la tradición y el dominio.

- Hipótesis 2. Es mayor la motivación del alumnado del profesorado que tiene un perfil centrado en la Innovación/ Disfrute que el del perfil centrado en la Tradición/Dominio.

\subsection{Participantes}

El estudio se realizó durante el curso 2015/2016 en 10 Escuelas de Música de Bizkaia. La selección de centros se realizó por conveniencia y también se vio determinada por el interés y disposición del profesorado para colaborar, obteniéndose una muestra en la que había diferencias en función del nivel socio-económico de las localidades donde están situados. Algunos centros son sólo Escuelas de Música y otros Escuelas de Música y Conservatorios, prestando también atención al tipo de música que ofertan ya que puede ser clásica, moderna o las dos.

Participaron 11 profesores y profesoras y el criterio fue que tuvieran formación y/o titulación de guitarra, al menos 3 años de experiencia en docencia en escuelas de música y diferentes formas de trabajar en el aula, datos que se obtuvieron a través de entrevistas personales durante el curso 2015/16. Se tuvieron en cuenta elementos como la paridad de género (profesoras y profesores) y diferente figura contractual (funcionarios, interinos, laborales, autónomos, eventuales...).

Tomaron parte en la investigación 195 alumnos y alumnas divididos en tres grupos de edad, de los cuales:

- El 11,8 \% fueron niños y niñas, de 7 a 11 años de edad.

- El 31,8\% fueron adolescentes, de entre 12 y 18 años de edad.

- El 36,4\% fueron adultos, a partir de 18 años sin límite de edad. 


\subsection{Instrumentos de medida}

La metodología se ajusta al principio de complementariedad de técnicas para dar respuesta a las diferentes preguntas de investigación planteadas (Creswell et al., 2003). Para ello, planteamos un estudio que combina técnicas de recogida y análisis de datos cuantitativos y cualitativos.

Para la evaluación del nivel de motivación se utilizó el cuestionario de Rey, Hidalgo y Espinosa (1989) que consta de 26 ítems y se fundamenta en 4 niveles distintos: nivel de interés, nivel de atracción y gusto por la asignatura, nivel de expectativas de interacción y nivel de interacción pedagógica. El sumatorio de las puntuaciones obtenidas en cada una de las escalas sitúa a los sujetos en niveles de motivación que van desde muy bajo hasta muy alto. Realizadas las pruebas de fiabilidad con el estadístico Alfa de Cronbach, se obtuvo un valor de 0.93969 .

En el caso del alumnado comprendido entre 7 y 11 años se utilizó el mismo cuestionario previamente validado en el que se suprimieron 4 ítems para adaptarlo al nivel de comprensión de estas edades.

Se ha optado por pruebas no paramétricas por ser muestras pequeñas que no cumplen criterios de normalidad y homocedasticidad necesarios para aplicar otras pruebas paramétricas como las ANOVAS. Las pruebas de Kolmogorov-Smirnov para estudiar la normalidad indican que la motivación no sigue una distribución normal sig $<0,05$ :

Tabla 2. Prueba de Kolmogorov-Smirnov para una muestra

\begin{tabular}{|l|l|r|}
\hline & & Motivación media \\
\hline $\mathrm{N}$ & & 195 \\
\hline Parámetros normalesa,b & Media & 3,9337 \\
\hline & Desviación estándar &, 41516 \\
\hline Máximas diferencias extremas & Absoluta &, 079 \\
\hline & Positivo &, 040 \\
\hline & Negativo &,- 079 \\
\hline Estadístico de prueba & &, 079 \\
\hline Sig. asintótica (bilateral) & &, $005 \mathrm{c}$ \\
\hline & & \\
\hline
\end{tabular}

a. La distribución de prueba es normal.

b. Se calcula a partir de datos.

c. Corrección de significación de Lilliefors.

Por otra parte, se grabaron en audio entrevistas semiestructuradas realizadas al profesorado con objeto de conocer su perfil educativo, en base a la clasificación realizada por Parkinson (2016). Los tópicos de dichas entrevistas fueron: formación musical, experiencia docente, objetivos, metodología, repertorio, lugar de los gustos del alumnado en la programación, motivación del alumnado y evaluación.

\subsection{Procedimiento}

Primeramente se realizaron una serie de reuniones con el profesorado participante en este estudio para concretar los términos de la investigación y concertar la forma de aplicar el cuestionario descrito.

En cuanto a las entrevistas realizadas al profesorado, una vez transcritas literalmente, se analizaron minuciosamente y se procedió a la identificación y clasificación de las diferentes categorías (Rodríguez, Gil y García, 1996) a través de la técnica de análisis de contenido, que supuso el agrupamiento de los datos en categorías de análisis. La forma de trabajo fue inductiva, a partir de los datos recabados, partiendo de la información particular para después llegar a un nivel de comprensión más global (Lorenzo, 2011). El proceso de categorización consistió en buscar temas comunes que facilitaran la obtención de resultados relacionados con los objetivos de la experiencia (Coffey \& Atkinson, 2003). Estas categorías, fueron inductivo-deductivas; inductivas, porque emergieron de las respuestas y deductivas, porque se apoyaron en la revisión de la literatura al respecto. Así, el primer análisis consistió en la lectura detallada de las entrevistas, dejando que aflorasen las voces de los participantes, haciendo una primera selección de los temas que iban surgiendo, para así construir la herramienta de análisis e interpretación de los datos a la que llamamos sistema categorial. A su vez, fue contrastado de forma deductiva con los principios metodológicos de los que parte la experiencia, generando un sistema categorial emergente.

La dirección del centro fue informada y, al en el caso del alumnado menor de edad, se solicitaron las autorizaciones a las familias. Se tuvieron en cuenta las normas éticas de confidencialidad, autorización de las familias y centros educativos y conservación segura de datos de las personas participantes. 


\section{Resultados}

\subsection{Caracterización del profesorado}

Se procedió a analizar las entrevistas del profesorado, con el fin de caracterizar la visión de su forma de trabajar en el aula, en función de los tipos ya descritos propuestos por Parkinson (2016). Los resultados obtenidos fueron los siguientes:

Los docentes 1, 3, 4, 5, 8 y 11 otorgan prioridad al hecho de que el estudiante esté a gusto. Su objetivo principal es que cuando esté en el aula disfrute, esté relajado y encuentre su espacio, perciba que su opinión y lo que siente es tenido en cuenta, "ya que una buena comunicación mejora la clase" y propicia la creación de un vínculo adecuado. También creen que la motivación es muy importante ya que es vital llegar al alumnado y desarrollar sus capacidades; otorgan importancia a que sean capaces de expresar y sentir que "la música tiene que salir del corazón para disfrutarla de verdad", "no importa la dificultad, una pequeña melodía puede ser alucinante." Intentan ser cercanos y cordiales para mejorar la clase, respetando las elecciones de los estudiantes, adaptando los materiales y realizando arreglos para que puedan tocar lo que les gusta y disfruten de la música. "Escuchar su opinión es muy importante". Coinciden en intentar ofrecer a su alumnado unos mínimos conceptos técnicos, pero intentan, principalmente, que este elija un repertorio con el que se sienta a gusto. Reconocen abiertamente que no tienen una estrategia determinada, ni una dirección definida sino que se limitan a ser ellos mismos.

Los docentes 2, 6, 7, 9 y 10 tienen como objetivo principal la destreza técnica y el manejo del instrumento; en general, no ven la necesidad de que se establezca un vínculo personal con el alumnado, porque creen que "si hay confianza te decepcionan y no estudian". Este profesorado no está de acuerdo en que el alumnado pueda elegir el repertorio y no adaptan los materiales para llegar a los estudiantes o facilitar el aprendizaje. Creen que el examen es una herramienta importante para el aprendizaje, para que "se lo tomen en serio y además a las familias les importa mucho la nota." Hablan positivamente de la motivación, pero con reservas, ya que debe ir acompañada de trabajo.

El profesor 6 trata de acercarse al alumnado para estar a gusto ambos, ya que piensa que una buena comunicación mejora la clase y considera que la motivación es la clave para aprender; no obstante, en su opinión, existe una barrera, que es la dificultad en la ejecución del instrumento, así que es muy importante estudiar en casa. Intenta potenciar en el alumnado el gusto por la música clásica, aunque reconoce que tiene que negociar. "Siempre les digo a mis alumnos y alumnas que lo que no conocen no les puede gustar, por eso insisto en que oigan música clásica."

Los docentes 7, 9 y 10 valoran bien establecer un vínculo con sus estudiantes, pero lo consideran una responsabilidad de estos: "crear un vínculo es fácil salvo que el alumnado tenga problemas de relación"; priorizan la técnica: "una buena relación con el alumnado favorece que encuentre su espacio y la clase sea más positiva pero para todo ello es importante una buena técnica" o manifiestan dudas: "establecer un vínculo personal con el alumnado es importante pero no demasiado porque pierde seriedad".

De los resultados anteriormente presentados se extrae que, de los 11 profesores y profesoras participantes en la investigación, 5 pertenecerían al grupo de tradición/dominio y 6 al de innovación y disfrute, tal y como se recoge en la Tabla 3:

Tabla 3. Clasificación del profesorado según edad, experiencia, estudios y filosofía sobre educación musical.

\begin{tabular}{|l|l|l|l|}
\hline Profesor & $\begin{array}{c}\text { Años experiencia } \\
\text { docente }\end{array}$ & \multicolumn{1}{|c|}{ Estudios } & $\begin{array}{c}\text { Filosofía sobre edu- } \\
\text { cación musical }\end{array}$ \\
\hline 1 & 13 & $\begin{array}{l}\text { Grado medio plan 66 } \\
\text { Grado superior otro instrumento }\end{array}$ & Innovación/disfrute \\
\hline 2. & 10 & $\begin{array}{l}\text { Grado medio plan 66 } \\
\text { Habilitación en música moderna. }\end{array}$ & Tradición/dominio \\
\hline 3 & 20 & Grado medio plan 66 & Innovación/disfrute \\
\hline 4 & 12 & $\begin{array}{l}\text { Grado medio plan LOGSE } \\
\text { Magisterio musical } \\
\text { Habilitación en música moderna. }\end{array}$ & Innovación/disfrute \\
\hline 5 & 14 & Grado medio plan LOGSE, sin terminar. & Innovación/disfrute \\
\hline 6 & 26 & Grado superior plan 66. & Tradición/dominio \\
\hline 7 & 25 & Grado superior plan 66. & Tradición/dominio \\
\hline 8 & 8 & Grado medio plan LOGSE sin terminar. & Innovación/disfrute \\
\hline 9 & 26 & $\begin{array}{l}\text { Grado Superior del plan 66 } \\
\text { Especialista en Jazz. }\end{array}$ & Tradición/dominio \\
\hline 10 & 25 & Grado superior plan 66 & Tradición/dominio \\
\hline
\end{tabular}




\begin{tabular}{|l|l|l|l|}
\hline 11 & 23 & $\begin{array}{l}\text { Grado Superior plan 66 } \\
\text { Magisterio musical } \\
\text { C.A.P. } \\
\text { Habilitación en música moderna. }\end{array}$ & Innovación/disfrute \\
\hline
\end{tabular}

Los resultados obtenidos permiten afirmar que se cumple la Hipótesis 1.

\subsection{Motivación del alumnado.}

En la tabla 4 se muestran los resultados de las puntuaciones medias de la motivación para cada grupo de edad de los participantes:

Tabla 4. Puntuación media de motivación del alumnado según edad

\begin{tabular}{|l|c|c|c|c|c|}
\hline \multirow{2}{*}{} & \multicolumn{5}{|c|}{ MOTIVACIÓN MEDIA } \\
\cline { 2 - 6 } & $\mathrm{N}$ & Media & $\begin{array}{c}\text { Mínimo } \\
\text { posible }\end{array}$ & $\begin{array}{c}\text { Máximo } \\
\text { posible }\end{array}$ & $\begin{array}{c}\text { Desviación } \\
\text { típica }\end{array}$ \\
\hline Niños & 23 & 4,01 & 1,00 & 5,00 & 0,34 \\
\hline Adolescentes & 101 & 3,90 & 1,00 & 5,00 & 0,45 \\
\hline Adultos & 71 & 3,96 & 1,00 & 5,00 & 0,38 \\
\hline Total & 195 & 3,93 & 1,00 & 5,00 & 0,42 \\
\hline
\end{tabular}

Una vez aplicado el estadístico de contraste a las medias obtenidas, la prueba de Kruskal Wallis (tabla 5), se comprueba que no hay diferencias significativas entre los tres grupos de edad en cuanto a la puntuación media de la motivación a un nivel de confianza del 5\%, tal y como puede comprobarse en la Tabla 5 :

Tabla 5: Diferencias en motivación entre los grupos de edad Prueba de Kruskal Wallis.

\begin{tabular}{|c|c|}
\hline Chi-cuadrado & 1,381 \\
\hline Gl & 2 \\
\hline Sig. asintótica & 0,501 \\
\hline
\end{tabular}

En cuanto a la relación entre la media de la motivación y la caracterización del profesorado, en la Tabla 6 se recoge la caracterización de cada uno de los educadores analizados y la media de motivación de su alumnado correspondiente:

Tabla 6: Filosofía del profesorado y media de motivación del alumnado

\begin{tabular}{|c|c|c|c|c|c|c|}
\hline & \multirow{2}{*}{$\begin{array}{c}\text { Filosofía sobre } \\
\text { educación musical }\end{array}$} & $\mathrm{N}$ & Media & $\begin{array}{c}\text { Mínimo } \\
\text { posible }\end{array}$ & $\begin{array}{c}\text { Máximo } \\
\text { posible }\end{array}$ & $\begin{array}{c}\text { Desviación } \\
\text { típica }\end{array}$ \\
\cline { 5 - 7 } Profesor 1 & $\begin{array}{c}\text { Innovación/ } \\
\text { disfrute }\end{array}$ & 11 & 3,85 & 1,00 & 5,00 & 0,43 \\
\hline Profesor 2 & $\begin{array}{c}\text { Tradición/ } \\
\text { dominio }\end{array}$ & 18 & 3,61 & 1,00 & 5,00 & 0,39 \\
\hline Profesor 3 & $\begin{array}{c}\text { Innovación/ } \\
\text { disfrute }\end{array}$ & 26 & 4,03 & 1,00 & 5,00 & 0,34 \\
\hline Profesor 4 & $\begin{array}{c}\text { Innovación/ } \\
\text { disfrute }\end{array}$ & 11 & 4,04 & 1,00 & 5,00 & 0,40 \\
\hline Profesor 5 & $\begin{array}{c}\text { Innovación/ } \\
\text { disfrute }\end{array}$ & 11 & 4,14 & 1,00 & 5,00 & 0,46 \\
\hline Profesor 6 & $\begin{array}{c}\text { Tradición } \\
\text { /dominio }\end{array}$ & 11 & 3,79 & 1,00 & 5,00 & 0,27 \\
\hline Profesor 7 & $\begin{array}{c}\text { Tradición/ } \\
\text { dominio }\end{array}$ & 12 & 3,85 & 1,00 & 5,00 & 0,36 \\
\hline
\end{tabular}




\begin{tabular}{|c|c|c|c|c|c|c|}
\hline Profesor 8 & $\begin{array}{c}\text { Innovación/ } \\
\text { disfrute }\end{array}$ & 15 & 3,86 & 1,00 & 5,00 & 0,37 \\
\hline Profesor 9 & $\begin{array}{c}\text { Tradición/ } \\
\text { dominio }\end{array}$ & 23 & 3,93 & 1,00 & 5,00 & 0,40 \\
\hline Profesor 10 & $\begin{array}{c}\text { Tradición/ } \\
\text { dominio }\end{array}$ & 21 & 3,92 & 1,00 & 5,00 & 0,43 \\
\hline Profesor 11 & $\begin{array}{c}\text { Innovación/ } \\
\text { disfrute }\end{array}$ & 36 & 4,07 & 1,00 & 5,00 & 0,46 \\
\hline \multicolumn{2}{|c|}{ Total } & 195 & 3,93 & 1,00 & 5,00 & 0,42 \\
\hline
\end{tabular}

En la Tabla 7 se muestran los resultados obtenidos para la muestra total de las puntuaciones medias de la motivación según el tipo de filosofía educativa del profesorado, mientras que en la Tabla 8 se analiza la correspondiente significatividad de las diferencias entre ambas puntuaciones, utilizando como estadístico de contraste la Prueba de U de Mann - Whitney:

Tabla 7. Puntuación media de motivación según tipo de profesor. Muestra total

\begin{tabular}{|l|l|c|c|c|c|c|}
\hline \multirow{2}{*}{} & \multicolumn{5}{|c|}{ MOTIVACIÓN MEDIA } \\
\cline { 3 - 7 } & $\mathrm{N}$ & Media & $\begin{array}{c}\text { Mínimo } \\
\text { posible }\end{array}$ & $\begin{array}{c}\text { Máximo } \\
\text { posible }\end{array}$ & $\begin{array}{c}\text { Desviación } \\
\text { típica }\end{array}$ \\
\hline \multirow{2}{*}{$\begin{array}{l}\text { Tipo pro- } \\
\text { fesorado }\end{array}$} & $\begin{array}{l}\text { Tradicional/ } \\
\text { Dominio }\end{array}$ & 85 & 3,83 & 1,00 & 5,00 & 0,40 \\
\cline { 2 - 7 } & $\begin{array}{l}\text { Innovador/ } \\
\text { Disfrute }\end{array}$ & 110 & 4,01 & 1,00 & 5,00 & 0,41 \\
\hline & Total & 195 & 3,93 & 1,00 & 5,00 & 0,42 \\
\hline
\end{tabular}

Tabla 8. Prueba de U-Mann. Diferencias de motivación según tipo de profesor

\begin{tabular}{|l|r|}
\hline & MOTIVACIÓN MEDIA \\
\hline U de Mann-Whitney & 3437,5 \\
\hline W de Wilcoxon & 7092,5 \\
\hline Z & $-3,168$ \\
\hline Sig. asintótica (bilateral) & 0,002 \\
\hline
\end{tabular}

Tal y como puede verse en la Tabla 8 se obtuvieron diferencias significativas entre la motivación del alumnado del profesorado tradicional/dominio y la del alumnado de docentes con marca de innovador/disfrute. Los resultados muestran valores más altos de motivación en el alumnado perteneciente al perfil de profesorado Innovador/Disfrute.

A continuación, se realizaron los mismos análisis para cada una de las edades del alumnado. Los resultados quedan recogidos en las Tablas 9, 10, 11, 12, 13 y 14.

Los resultados de las puntuaciones medias de la motivación para la muestra de niños y niñas y la correspondiente significatividad de las diferencias utilizando como estadístico de contraste la Prueba de U de Mann - Whitney quedan recogidos a continuación en las Tablas 9 y 10:

Tabla 9. Puntuación media de motivación según tipo de profesor. Muestra niños/niñas

\begin{tabular}{|l|l|c|c|c|c|c|}
\hline \multirow{2}{*}{} & \multicolumn{5}{|c|}{ MOTIVACIÓN MEDIA } \\
\cline { 3 - 6 } & $\mathrm{N}$ & Media & $\begin{array}{c}\text { Mínimo } \\
\text { posible }\end{array}$ & $\begin{array}{c}\text { Máximo } \\
\text { posible }\end{array}$ & $\begin{array}{c}\text { Desviación } \\
\text { típica }\end{array}$ \\
\hline \multirow{2}{*}{$\begin{array}{l}\text { Tipo } \\
\text { profesorado }\end{array}$} & $\begin{array}{l}\text { Tradicional/ } \\
\text { Dominio }\end{array}$ & 10 & 3,84 & 1,00 & 5,00 & 0,33 \\
\cline { 2 - 6 } & $\begin{array}{l}\text { Innovador/ } \\
\text { Disfrute }\end{array}$ & 13 & 4,14 & 1,00 & 5,00 & 0,29 \\
\hline & Total & 23 & 4,01 & 1,00 & 5,00 & 0,34 \\
\hline
\end{tabular}


Tabla 10. Prueba de U-Mann. Diferencias de motivación según tipo de profesor. Muestra niños/niñas

\begin{tabular}{|l|r|}
\hline & MOTIVACIÓN MEDIA \\
\hline U de Mann-Whitney & 30 \\
\hline W de Wilcoxon & 85 \\
\hline$Z$ & $-2,174$ \\
\hline Sig. asintótica (bilateral) & 0,030 \\
\hline
\end{tabular}

La Tabla 10 muestra que había diferencias significativas entre la motivación del alumnado del profesorado tradicional/dominio y la del alumnado de docentes con marca de innovador/disfrute. Los resultados también en este caso son valores más altos de motivación en el alumnado perteneciente al perfil de profesorado Innovador/Disfrute.

Los resultados de las puntuaciones medias de la motivación obtenidos por adolescentes y su correspondiente significatividad de las diferencias utilizando como estadístico de contraste la Prueba de U de Mann - Whitney se pueden ver en las Tablas 11 y 12 :

Tabla 11. Puntuación media de motivación según tipo de profesor. Muestra adolescentes

\begin{tabular}{|l|l|c|c|c|c|c|}
\hline \multirow{2}{*}{} & \multicolumn{5}{|c|}{ MOTIVACIÓN MEDIA } \\
\cline { 3 - 7 } & $\mathrm{N}$ & Media & $\begin{array}{c}\text { Mínimo } \\
\text { posible }\end{array}$ & $\begin{array}{c}\text { Máximo } \\
\text { posible }\end{array}$ & $\begin{array}{c}\text { Desviación } \\
\text { típica }\end{array}$ \\
\hline \multirow{2}{*}{$\begin{array}{l}\text { Tipo } \\
\text { profesorado }\end{array}$} & $\begin{array}{l}\text { Tradicional/ } \\
\text { Dominio }\end{array}$ & 37 & 3,77 & 1,00 & 5,00 & 0,42 \\
\cline { 2 - 7 } & $\begin{array}{l}\text { Innovador/ } \\
\text { Disfrute }\end{array}$ & 64 & 3,97 & 1,00 & 5,00 & 0,45 \\
\hline & Total & 101 & 3,90 & 1,00 & 5,00 & 0,45 \\
\hline
\end{tabular}

Tabla 12. Prueba de U-Mann. Diferencias de motivación según tipo de profesor. Muestra adolescentes

\begin{tabular}{|l|l|}
\hline & MOTIVACIÓN MEDIA \\
\hline U de Mann-Whitney & 867 \\
\hline W de Wilcoxon & 1570 \\
\hline Z & $-2,236$ \\
\hline Sig. asintótica (bilateral) & 0,025 \\
\hline
\end{tabular}

También en este caso la Tabla 12 muestra diferencias significativas entre la motivación del alumnado del profesorado tradicional/dominio y la del alumnado de docentes con marca de innovador/disfrute. Igualmente, son los valores más altos de motivación los del alumnado perteneciente al perfil de profesorado Innovador/Disfrute.

Finalmente, los resultados referentes al colectivo de adultos quedan recogidos en las Tablas 13 y 14:

Tabla 13. Puntuación media de motivación según tipo de profesor. Muestra adultos

\begin{tabular}{|l|l|c|c|c|c|c|}
\hline \multirow{2}{*}{} & \multicolumn{5}{|c|}{ MOTIVACIÓN MEDIA } \\
\cline { 3 - 7 } & $\mathrm{N}$ & Media & $\begin{array}{c}\text { Mínimo } \\
\text { posible }\end{array}$ & $\begin{array}{c}\text { Máximo } \\
\text { posible }\end{array}$ & $\begin{array}{c}\text { Desviación } \\
\text { típica }\end{array}$ \\
\hline \multirow{2}{*}{$\begin{array}{l}\text { Tipo } \\
\text { profesorado }\end{array}$} & $\begin{array}{l}\text { Tradicional/ } \\
\text { Dominio }\end{array}$ & 38 & 3,89 & 1,00 & 5,00 & 0,39 \\
\cline { 2 - 7 } & $\begin{array}{l}\text { Innovador/ } \\
\text { Disfrute }\end{array}$ & 33 & 4,05 & 1,00 & 5,00 & 0,37 \\
\hline & Total & 71 & 3,96 & 1,00 & 5,00 & 0,38 \\
\hline
\end{tabular}


Tabla 14. Prueba de U-Mann. Diferencias de motivación según tipo de profesor. Muestra adultos

\begin{tabular}{|l|r|}
\hline & \multicolumn{1}{|c|}{ MOTIVACIÓN MEDIA } \\
\hline U de Mann-Whitney & 472 \\
\hline W de Wilcoxon & 1213 \\
\hline$Z$ & $-1,788$ \\
\hline Sig. asintótica (bilateral) & 0,074 \\
\hline
\end{tabular}

En este último caso, no se obtuvieron diferencias significativas entre la motivación del alumnado y el perfil del profesorado.

En consecuencia, los resultados obtenidos nos permiten afirmar que se cumple la Hipótesis 2, salvo para el caso del alumnado adulto.

\section{Conclusiones}

En esta investigación, nos hemos interesado por la posible relación que pudiera tener la motivación en las Escuelas de música con la forma de entender la educación los docentes y el modo de relacionarse con su alumnado que se deriva de esta.

Los resultados obtenidos en las entrevistas al profesorado, evidencian diferencias en su forma de trabajar en el aula, que parecen responder a las características de los perfiles descritos en el marco teórico (Parkinson, 2016). En este sentido queremos subrayar, como ya se ha indicado, que el modelo no pretende polarizar perfiles de profesorado, sino sugerir elementos para una práctica docente reflexiva.

La mayoría del profesorado al que hemos atribuido un perfil tradicional en función de sus respuestas a la entrevista, se caracteriza por haber realizado estudios superiores en el instrumento, tener más años de experiencia docente (véase Tabla 3 ) y por incidir más en la destreza técnica del alumnado. Hay que subrayar que el profesorado con más años de experiencia docente, comenzó a trabajar en conservatorios que se convirtieron en Escuelas de Música y quizá no sea sencillo adaptarse al cambio de mentalidad y necesidades de este tipo de centros (Morant, 2013). Es posible que la falta de formación pedagógica haga que el profesorado fije su atención en la técnica y no atienda aspectos más personales y relacionales, que podrían ser claves en la motivación del alumnado de estos centros (López, 2016; Torrado y Pozo, 2008; Widad, 2013). Por su parte, el profesorado más joven, con mayor formación en pedagogía, que empezó a trabajar en escuelas de música, en general responde al perfil innovador.

En cuanto a los resultados obtenidos en relación a la Hipótesis 2, parecen corroborarla salvo en el caso de los adultos.

En efecto, el alumnado de los docentes innovadores enfocados en el disfrute en el aprendizaje de la música (Parkinson, 2016), presenta un nivel de motivación más alto y, se caracteriza principalmente por tener una relación más próxima y afectiva con sus educadores; esto confirma resultados anteriores en otros contextos educativos (Madariaga y Arriaga, 2011; Campayo y Cabedo, 2018), en el sentido de que la motivación en educación musical está relacionada con la interacción entre profesorado y alumnado, principalmente cuando se ofrece confianza y un sentimiento de apoyo. Además, se han podido precisar estrategias concretas vinculadas al disfrute y valoración del alumnado, principales características del modelo innovador, las cuales a su vez se han mencionado en investigaciones previas. Así, los índices más elevados de motivación coinciden con un profesorado que cree en la necesidad de establecer un vínculo con el alumnado, dialoga, otorga importancia a su opinión y su sentir, e intenta adaptar los materiales para que pueda interpretar y disfrutar la música. También trata de que dicho alumnado se encuentre relajado (Cobo, 2014), buscando una relación personal (Burnett, 1999), invirtiendo tiempo en conocerle para adaptarse a sus necesidades y proporcionarle un ambiente de aprendizaje acogedor y atractivo en el que el alumnado sienta que es valorado (Codgill, 2015) y teniendo en cuenta sus intereses a la hora de elegir un repertorio apropiado y adaptado para fomentar la habilidad y el interés (Cain, 2013; Codgill, 2015; Jorquera, 2002; Renwick y McPherson, 2002).

Por el contrario, cuando el profesorado otorga excesiva importancia a la destreza técnica (Parkinson, 2016), no facilita que el alumnado elija su repertorio, incide en su correcta ejecución, características del profesorado tradicional y además, no adapta los materiales y concibe que el examen es una herramienta que invita al alumnado a estudiar, los resultados de la motivación del alumnado tienen un nivel más bajo.

En el caso del alumnado adulto también está motivado pero, la filosofía educativa y la forma de vincularse afectivamente con el profesorado que de esta se deriva, no parece afectarle igual que al resto, seguramente por su mayor madurez. De hecho, es posible que tenga otro tipo de necesidades no tan relacionadas con la confianza del profesorado y el clima de aula, además de tener más libertad y autonomía para cambiarse de profesor/a si lo viese necesario.

Respecto a la elección del repertorio por el alumnado, el profesorado de la muestra lo facilita, pero no parece que el objetivo sea un estudio profundo del mismo, sino una forma de disfrutar de la música, lo que conlleva que la motivación resultante sea más elevada (Asmus, 1993).

En esta dirección concluyen igualmente Evans, McPherson y Davidson (2013), quienes afirman que es necesario que los docentes se aseguren de que son cálidos y accesibles para promover la autonomía del alumnado y proporcio- 
nar situaciones en los que sienta que puede aportar algo a la planificación de sus estudios, además de utilizar niveles de desafío óptimos para mejorar la motivación. Dado que las necesidades de los estudiantes de instrumento son muy variadas y es el profesorado el que precisa identificar y dar respuesta a las mismas (Cain, 2013), es importante incidir en la necesidad de impulsar y facilitar cursos de formación en los que, además, de aspectos pedagógicos se incluyan también conceptos de desarrollo de la persona y de habilidades sociales para interactuar con el alumnado. En este sentido, sería muy beneficioso incluir en la formación del profesorado de las escuelas de música contenidos y prácticas que incidan en la importancia de conocer y explorar las necesidades de su alumnado, las razones que les motivan para involucrarse en el aprendizaje musical, así como en la creación un ambiente acogedor y alentador. Esta forma de acercarse al alumnado le ayudará a desarrollar estrategias apropiadas que motiven al alumnado para participar en actividades musicales durante toda su vida.

\section{Referencias bibliográficas}

Arriaga, C., \& Madariaga, J. M. (2014). Is the perception of music related to musical motivation in school? Music Education Research, 16(4), 375-96. https://doi.org/10.1080/14613808.2013.847074

Asmus, E.P. (1993). Motivation in music teaching and learning. Paper presented at the Indiana Symposium on Research in Social Psychology of Music. Indiana University.

Austin, J.R. Renwick, J., \& McPherson, G. E. (2006). Developing Motivation. En G. E. McPherson (Ed.), The Child as Musician: A Handbook of Musical Development (pp. 213-238). Oxford, England: Oxford University Press. doi:10.1093/acprof:oso/9780198530329.003.0011.

Burnett, P. C. (1999). Children's self-talk and academic self-concepts. Educational Psychology in Practice, 15(3), $195-200$.

Burwell, K., Pickup, D. \& Young, V. (2003). Areas of study and teaching: a case study research project. Music Education Research, 5(2).139-155.

Cain, T. (2013). Investigación-acción en Educación Musical. En M. Díaz \& A. Giráldez (Coords.), Investigación Cualitativa en Educación Musical (pp. 57-75). Barcelona: Graó.

Campayo, E. \& Cabedo, A. (2018). El rol de padres, profesores y compañeros en la motivación de estudiantes de enseñanzas elementales de piano en conservatorios: un estudio de caso múltiple. Revista Internacional de Educación Musical, 6, 43-51. https://doi.org/10.1177/230748411800600104

Cobo, A. (2014). La gestión de las emociones en el aula: ¿técnica o arte? Un puente emocional necesario. Aula de Secundaria, 8, 25-28.

Cogdill, S. H. (2015). Applying research in motivation and learning to music education: What the experts say. Update: Applications of Research in Music Education, 33(2), 49-57.

Creswell, J., Plano, V., Gutmann, M. y Hanson, W. (2003). Advanced mixed methods research designs. In Tashakkoei y Teddlie (Eds), Handbook of mixed methods in social and behavioral research (209-240). London: SAGO publications.

De Alba, B. (2015). Las Escuelas de música del País Vasco. Análisis de una realidad educativa .Tesis doctoral. Universidad del País Vasco. Recuperado de: https://addi.ehu.es/bitstream/handle/10810/17750/9082-379-8-DeAlbaTH-INet.pdf?sequence=1

De Bézenac, C., \& Swindells, R. (2009). No pain, no gain? Motivation and self-regulation in music learning. International Journal of Education and the Arts, 10(16), 1-33.

Evans, P., McPherson, G. E., \& Davidson, J. W. (2013). The role of psychological needs in ceasing music and music learning activities. Psychology of Music, 41(5), 600-619.

Evans, P. (2015). Self-determination theory: An approach to motivation in music education. Musicae Scientiae, 19(1), 65-83. https://doi.org/10.1177/1029864914568044

Gobierno Vasco (1996). Instrucciones sobre la organización del curso en los centros de enseñanzas musicales. Vitoria-Gasteiz: Gobierno Vasco.

Hallam, S. (2002). Musical Motivation: Towards a Model Synthesising the Research. Music Education Research, 4(2), $225-244$. doi:10.1080/1461380022000011939

Hallam,S. \& Creech, A. (2010). Learning to play an instrument. En S. Hallam \& A. Creech (Eds.), Music education in the 21st century in the United Kingdom: Achievements, analysis and aspirations (pp. 85-104). London: Institute of Education.

Jorquera, C. (2002). ¿Existe una didáctica del instrumento musical? Revista Electrónica de LEEME, 9, 1-12. Recuperado de: https://ojs.uv.es/index.php/LEEME/article/view/9731

López, F. (2016). La teoría de metas de logro como factor de motivación. Un análisis en las clases instrumentales de conservatorio. Revista Electrónica de LEEME, 37, 35-50. Recuperado de: http://musica.rediris.es/leeme/revista/lopez16.pdf

Lorenzo, Oswaldo. 2011. "Análisis cualitativo de textos sobre multi e interculturalidad”. DEDiCA. Revista de Educação e Humanidades.Vol.1: 535-546.

Macworth-Young, L. (1990). Pupil centred learning in piano lessons: an evaluated action-research programme focusing on the psychology of the individual. Psychology of Music, 18, 73-86. doi: 10.1177/0305735690181006.

McPhail, G. J. (2010). Crossing boundaries: sharing concepts of music teaching from classroom to studio. Music Education Research, 12 (1), 33-45.

McPherson, G. E. \& McCormick, J. (1999). Motivational and self-regulated learning components of musical practice. Bulletin of the Council for Research in Music Education, 141, 98-102. 
McPherson, G. E. \& O’Neill, S. A. (2010). Students motivation to study music as compared to other school subjects: A comparison of eight countries. Research Studies in Music Education, 32(2), 101-137.

Madariaga, J.M., \& Arriaga, C. (2011). Análisis de la práctica educativa del profesorado de música y su relación con la motivación del alumnado. Cultura y Educación, 23 (3), 463-476. doi:10.1174/113564011797330234.

Morant, R. (2013). Perspectivas docentes de las escuelas de música de las sociedades musicales valencianas: historia, presente y futuro. Revista Electrónica de LEEME, 34, 18-35. Recuperado de: https://dialnet.unirioja.es/servlet/tesis?codigo=116293

Parkinson,T. (2016). Mastery, enjoyment, tradition and innovation: A reflective practice model for instrumental and vocal teachers. International Journal of Music Education, 34(3), 352-368. doi: 10.1177/0255761414563196.

Pozo, J. I. , Bautista, A., \&Torrado, J. A. (2008). El aprendizaje y la enseñanza de la interpretación musical: cambiando las concepciones y las prácticas. Cultura y Educación, 20(1), 5-15.

Renwick, J. \& McPherson, G. E. (2002). Interest and choice. Student-selected repertoire and its effect on practising behaviour. British Journal of Music Education, 19(2), 173-188.

Rey, J. M., Hidalgo, E. \& Espinosa, C. (1989). La motivación en la Escuela: cuestionario para su análisis. Málaga: Agora.

Rodríguez, G., Gil, J. \& García, E. (1996). Metodología de la investigación cualitativa. Málaga: Aljibe.

Torrado, J.A., \& Pozo, J.L. (2008). Metas y estrategias para una práctica constructiva en la enseñanza instrumental. Cultura y Educación, 20(1), 35-48.

Triptana, S. (2016). Estrategias de motivación durante el aprendizaje instrumental. Revista Internacional de Educación Musical, 4, 25-32.

West, C. (2013). Motivating music students: A review of the literature. Update: Applications of research in music education, 31(2), 11-19. doi.org/10.1177/8755123312473611.

Widad, S. (2013). Relación docente-discente: gestión de poder y relación humana. Actas del $4^{\circ}$ taller "Ele e interculturalidad". Centro virtual del Instituto Cervantes de Orán. Recuperado de: https://cvc.cervantes.es/ensenanza/biblioteca_ele/publicaciones_centros/PDF/oran_2013/18_widad.pdf 